\title{
ReaR
}

ISNN 19894090

Revista electrónica de AnestesiaR

LECTURA CRÍTICA DE ARTÍCULO

Marzo 2020

\section{Mejorando la seguridad del paciente: los medicamentos en la bandeja de anestesia.}

Artículo original: Almghairbi DS, Sharp L, Griffiths R, Evley R, Gupta S, et al. An observational feasibility study of a new anaesthesia drug storage tray. Anaesthesia. 2018 Mar;73(3):356-364. doi: 10.1111/anae.14187. Epub 2018 Jan 3. (

Power Esteban M, Lema Tomé M, Cabrerizo Torrente $P$

Hospital General Universitario Gregorio Marañón, Madrid.

\section{Resumen}

Los errores de medicación en anestesia, siguen siendo una causa grave de daño iatrogénico. Presentamos un estudio en el que se evalúa el potencial impacto de seguridad del uso de una bandeja con códigos de colores para la preparación y el almacenamiento de medicamentos anestésicos.

\section{Introducción}

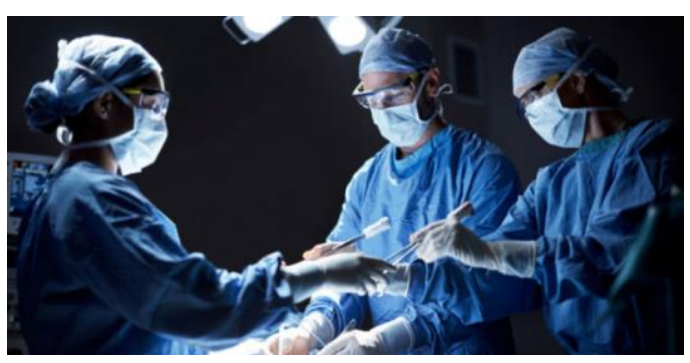

Los errores de medicación en anestesia, siguen siendo una causa grave de daño iatrogénico. Presentamos un estudio en el que se evalúa el potencial impacto de seguridad del uso de una bandeja con códigos de colores para la preparación y el almacenamiento de medicamentos anestésicos.

\section{Métodos}

Se trata de un estudio cualitativo observacional multicéntrico, que engloba tres instituciones de Inglaterra (a las que denominan $\mathrm{A}, \mathrm{B}$ y $\mathrm{C}$ ). Durante seis meses, tres investigadores observaron 30 preparaciones de medicación, 10 de las cuales involucraron las bandejas estándar en la práctica normal y 20 incluyeron las nuevas 'bandejas Rainbow'.

Se consideró como práctica normal en las tres instituciones, que los anestesistas recojan y transporten sus medicamentos preparados en una o más bandejas desechables,

unicompartimentales y de un solo color; mientras que las bandejas Rainbow constan de tres bandejas, una para medicamentos que no son de emergencia, otra para medicamentos de emergencia $y$ otra para anestésicos locales.

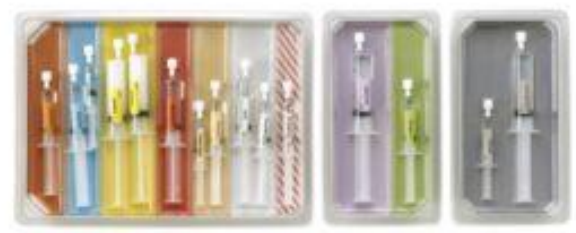

Figura 1. Bandeja 'Rainbow'

Presentaron las bandejas Rainbow a cada anestesista tras una observación inicial con las bandejas estándar. Dos semanas después, llevaron cabo una segunda inspección de la preparación de 
medicación usando las bandejas Rainbow, e inmediatamente después realizaron un total de 20 entrevistas semiestructuradas (apéndice 1).

\section{Resultados}

La práctica estándar en los tres sitios era preparar las jeringas antes de que el paciente entrara en el antequirófano, en una bandeja de papel gris, desechable; posteriormente 10 de 20 anestesistas leyeron en voz alta la etiqueta del medicamento, antes de cargar el mismo. Una vez preparado, todos los anestesistas etiquetaron la jeringa, pero no confirmaron previa administración, que el medicamento en la jeringa correspondía al medicamento de la ampolla desechada.

Cuando los medicamentos eran revisados por otra persona, siempre lo hacía un anestesista y en una de las instituciones no observaron ninguna doble verificación. En las instituciones A y $\mathrm{C}$, las ampollas vacías se guardaron hasta el final de la operación; mientras que en el B se desecharon después de la preparación. En las tres instituciones, el anestesista sostuvo las jeringas en la mano durante la inducción; sin doble verificación antes de su administración. No pudieron determinar con qué precisión el anestesista verificó cada jeringa antes de la administración de su contenido.

Observaron que los anestesistas a veces usaban más de una bandeja para separar las jeringas que contenían medicamentos de emergencia de medicamentos de inducción. Las bandejas con jeringas de fármacos de inducción se colocaban en la máquina de anestesia durante la inducción, mientras que las bandejas con jeringas de emergencia se dejaban donde se habían preparado.
Descubrieron que no había una práctica "estándar" para la preparación de medicamentos.

Los beneficios percibidos de las bandejas grises fueron la facilidad de uso, el tamaño y el coste, junto con la capacidad de separar los múltiples fármacos utilizados durante la anestesia. Sin embargo, se observó que dificultaba la lectura de las etiquetas, se usaban más jeringas y se reconocía por unanimidad que una bandeja llena podía provocar errores, particularmente cuando las ampollas usadas se conservaban en la bandeja.

La introducción de las bandejas Rainbow no pareció cambiar la forma en que se preparaba la medicación para la anestesia. Sin embargo, sí influyó en el almacenamiento de las jeringas mientras estaba en uso, de acuerdo con su propósito previsto.

Una vez que un medicamento se cargó y se etiquetó, se colocó en el compartimento codificado por colores dentro de la bandeja Rainbow; tanto para los medicamentos de la inducción como para los de emergencia.

Estas eran colocadas en posiciones similares a las bandejas grises en la sala, pero ya no sostenían varias jeringas durante la inducción, sino que retiraban las jeringas de la bandeja individualmente y volvían a revisar la etiqueta antes de la administración.

Las bandejas Rainbow se consideraron fáciles de usar y parecían ayudar a la identificación de fármacos mediante la compartimentación codificada por colores, que reforzaba el etiquetado individual de las jeringas.

También parecía aumentar la conciencia de los anestesistas sobre el riesgo de errores de medicación. Sin embargo, algunos comentaron que eran 
demasiado grandes y/o sus compartimentos demasiado pequeños.

Varios anestesistas identificaron un riesgo de intercambio de jeringas utilizando la bandeja Rainbow, debido a una compartimentación incorrecta no reconocida.

La mayoría de los participantes estaban preocupados por el coste de las bandejas Rainbow.

\section{Discusión}

Los anestesistas pensaban que su sistema actual de almacenamiento de medicamentos era fácil de usar, de bajo costo y manejable, pero tenía el riesgo potencial de errores de intercambio de jeringas, especialmente cuando las bandejas estaban llenas. Aceptaron rápidamente las bandejas Rainbow, prefiriéndolas a las estándar y pensaron que tenían el potencial de reducir los errores de medicación.

No encontraron una práctica "estándar" para la preparación de fármacos, a pesar de ser un componente fundamental de la práctica anestésica segura.

La preparación y el manejo del medicamento es una tarea repetida, de baja variabilidad y de alto riesgo. La literatura publicada describe la preparación del fármaco como una actividad clínica potencialmente de alto riesgo, y varios factores podrían reducir la posibilidad de error. Sin embargo, todavía no existe un consenso definitivo sobre el mejor método para preparar medicamentos, ya que ningún sistema que involucre a humanos estará libre de errores.

La introducción de la bandeja Rainbow pareció tener beneficios más allá del diseño en sí, y los participantes identificaron una mayor conciencia sobre la seguridad de los medicamentos.

Las bandejas de cartón siguen disponibles en las tres instituciones y, sin embargo, los anestesistas continúan eligiendo separar los anestésicos locales, de emergencia y de rutina con el sistema de bandejas Rainbow.

Las encuestas reflejaron inquietudes sobre el tamaño de la bandeja Rainbow y el de los compartimentos, lo que sugiere que es necesario un nuevo rediseño de ésta.

Otro hallazgo inesperado fue que algunos anestesistas continuaron "tapando" las jeringas con agujas, a pesar de que esta práctica no se recomienda y que las bandejas Rainbow se diseñaron deliberadamente para no acomodar una jeringa con aguja.

Se necesita más investigación para determinar las mejores estrategias para asegurar el uso continuo de las bandejas Rainbow, y su efecto sobre la prevalencia en los errores de medicación.

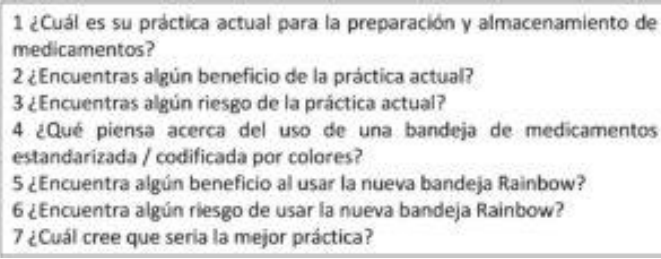

Apéndice 1 - Preguntas de la entrevista

\section{Comentario}

Los errores medicación en anestesia representan una amenaza para la seguridad del paciente, así como una causa importante de morbilidad $y$ mortalidad [1].

La administración de medicación en el entorno perioperatorio presenta desafíos para la seguridad del paciente en comparación con otros entornos hospitalarios[2]; a diferencia de otros entornos, la administración de medicación perioperatoria, hoy en día a 
menudo pasa por alto los controles de seguridad estándar como son la prescripción, la aprobación de farmacia y los múltiples chequeos de enfermería en el momento de su administración; a lo que se une la naturaleza altamente estresante y sensible al tiempo del cuidado de un quirófano; factores que pueden contribuir a tasas de errores de medicación más altas [3].

La tasa de errores de administración de medicamentos de anestesia no se conoce con certeza; las tasas de error basadas en la auto información facilitada varían desde $0.11 \%$ [4] hasta $0.75 \%$ [5,6]; otros estudios hablan de errores de medicación o eventos adversos en aproximadamente una de cada 20 administraciones de fármacos perioperatorios. [7] Independientemente de la verdadera tasa de error, su reducción debe ser una prioridad para todos los anestesistas. [3]

La mayoría de los países desarrollados han intentado mejorar la prescripción, preparación y administración de medicamentos para ayudar a reducir la dificultad inherente de este proceso [9], pero sigue siendo una causa grave de daño iatrogénico $[8,10]$. Existen diversas prácticas en anestesia, que intentan reducir los errores de medicación, entre los que encontramos las etiquetas con código de colores y preimpresas con el nombre del medicamento, las restricciones $\mathrm{y}$ normas en el almacén de medicamentos en el carro de anestesia, las jeringas precargadas, el sistema de doble verificación; no obstante, todo esto parece ser insuficiente, ya que determinados estudios mantienen que la tasa de errores se ha mantenido sin cambios en los últimos años. [4, 6, 11,12]. Cabe recordar, que pese a las diferentes medidas que instauremos para reducir la tasa de errores, existe un factor inherente a la administración de fármacos, que es el factor humano, imposible de eliminar, por ello es importante investigar e instaurar nuevas medidas barrera en adición a las actuales, para disminuir en la medida de lo posible al máximo los errores.

El presente estudio, presenta una opción más, como potencial herramienta para evitar errores en la administración de medicación perioperatoria. Esta novedosa medida que supone la bandeja 'Rainbow' así como los resultados descritos, parecen alentadores e incitan a su instauración y uso dentro de la practica anestésica.

\section{Bibliografía}

1. Fasting S, Gisvold SE. Adverse drug errors in anesthesia, and the impact of coloured syringe labels. Can J Anaesth 2000; 47: 1060e7 (ubMed)

2. Merry AF, Webster CS, Hannam J, Mitchell SJ, Henderson R, Reid P, et al: Multimodal system designed to reduce errors in recording and administration of drugs in anaesthesia: Prospective randomised clinical evaluation. BMJ 2011; 343:d5543 (PubMed)

3. Bowdle T. A.et al. Facilitated selfreported anaesthetic medication errors before and after implementation of a safety bundle and barcode-based safety system. British Journal of Anaesthesia, 121 (6): 1338e1345 (2018) (HTML)

4. Webster CS, Merry AF, Larsson L, McGrath KA, Weller J. The frequency and nature of drug administration error during anaesthesia. Anaesth Intensive Care 2001; 29:494e500

5. Llewellyn RL, Gordon PC, Wheatcroft D, Lines D, Reed A, Butt AD et al. Drug administration errors: a prospective survey from three South African teaching hospitals. Anaesth Intensive Care 2009; 37: 93e8 (PubMed)

6. Nanji KC, Patel A, Shaikh S, Seger DL, Bates DW. Evaluation of perioperative medication errors and adverse drug events. Anesthesiology 2016; 124: 25-34. (ubMed)

7. McClelland L, Holland J, Lomas J, Redfern N, Plunkett E. A national survey of the effects of fatigue on trainees in anaesthesia in the UK. 
Anaesthesia 2017; 72: 1069-77. (PubMed)

8. Murianni L, Marano C. Building a safer NHS for patient. Improving medication safety. Italian Journal of Public Health 2012; 2: 3-4. (PDF)

9. World Health Organisation. WHO launches global effort to halve medication-related errors in 5 years. 2017.

http://www.who.int/mediacentre/news/ releases $/ 2017 /$ medicationrelatederrors/en/ (accessed 25/10/2017).

10. Cooper L, DiGiovanni N, Schultz L, Taylor AM, Nossaman B. Influences observed on incidence and reporting of medication errors in anesthesia. Canadian Journal of Anesthesia 2012; 59: 562-70. ( $\underline{\text { PubMed })}$

11. Chopra V, Bovill J, Spierdijk J. Accidents, near accidents and complications during anaesthesia A retrospective analysis of a 10-year period in a teaching hospital. Anaesthesia 1990; 45: 3-6. (ubMed)

Correspondencia al autor

Mercedes Power Esteban

poweresteban@gmail.com

MIR Anestesiología y Reanimación.

Hospital General Universitario Gregorio Marañón, Madrid.

Maria Lema Tomé

maria.lematome@gmail.com

FEA Anestesiología y Reanimación.

Hospital General Universitario Gregorio Marañón, Madrid.

Aceptado para el blog en octubre de 2019 